\title{
Pharmaceutical Sales Representatives and Patient Safety
}

\author{
Barbara Mintzes, $P h D^{7}$, Joel Lexchin, $M D^{2}$, Michael S. Wilkes, $M D^{3}$, Marie-Dominique Beaulieu, $M D^{4}$, \\ Ellen Reynolds, $B A^{5}$, Jason Sutherland, $P h D^{5}$, and Geneviève Durrieu, PharmD, $P h D^{6}$ \\ ${ }^{1}$ School of Population and Public Health, University of British Columbia, Vancouver, British Columbia, Canada; ${ }^{2}$ York University, Ontario, \\ Canada; ${ }^{3}$ University of California Davis, Davis, USA; ${ }^{4}$ University of Montreal, Quebec, Canada; ${ }^{5}$ University of British Columbia, Vancouver, \\ Canada; ${ }^{6}$ University of Toulouse, Toulouse, France.
}

J Gen Intern Med 28(11):1395

DOI: $10.1007 / \mathrm{s} 11606-013-2563-5$

() Society of General Internal Medicine 2013

Authors' reply - In his letter concerning our study on the information that pharmaceutical sales representatives provided to primary care physicians, ${ }^{1}$ Donald Light highlights the harm that can occur to patients when information on serious harm is omitted, and characterizes such omissions as an untruthful use of commercial free speech. ${ }^{2}$ We agree that the mention only of drug benefits is inaccurate, because it provides a misleading impression of the full range of documented effects on patient health.

However, our results raise an additional health concern. Promotional messages draw attention to specific products, and may bias care simply because these drugs are uppermost in a physician's mind. Where samples are allowed, they provide an additional incentive to start patients on the drug. To quote one of the participating doctors, "... physicians are prescribing the drugs because it's convenient... So we've got samples, it's convenient, the information seems reasonable, and we prescribe them." This convenience may seem helpful or at worst innocuous, and patients often appreciate the gift of a sample, but if this leads to prescriptions for drugs that are less safe or effective than alternatives, patient health may suffer.

Rosuvastatin was the most frequently promoted drug in our study and is the highest potency statin available. The drug's effectiveness in lowering cholesterol was a frequent claim. This is true, but misleading in terms of patient health. High potency statins are associated with higher rates of acute kidney injury than lower potency statins. ${ }^{3}$ A systematic review comparing statins that reduce low-density lipoprotein (LDL) by $\geq 50 \%$ with lower dose or potency products found no net benefit in terms of survival or serious morbidity. ${ }^{4}$
Escitalopram (Lexapro), a serotonin-specific reuptake inhibitor (SSRI) antidepressant, was number two, and duloxetine, a serotonin-norepinephrine reuptake inhibitor (SNRI), number five, on our list of most promoted products. The effectiveness of both has been exaggerated through publication bias. ${ }^{5}$ Escitalopram is an isomer of citalopram, costlier but no more effective. Like citalopram, it is subject to regulatory warnings for torsades de pointes. Duloxetine, also without efficacy advantages, has risks of dose-dependent hypertension and liver injury. Claims included "less side effects" or "good tolerance, good effectiveness, from the first week onward."

These are but a few examples of the need for caution. Sales representatives are personable and provide easily accessible information. The downside of allowing this to guide prescribing choice may not only be extra cost, but also poorer quality care.

Corresponding Author: Barbara Mintzes, $\mathrm{PhD}$; School of Population and Public Health, University of British Columbia, Vancouver, British Columbia V6T 1Z3, Canada (e-mail: Barbara.mintzes@ti.ubc.ca).

\section{REFERENCES}

1. Mintzes B, Lexchin $J$, Sutherland $J$, et al. Pharmaceutical sales representatives and patient safety: a comparative prospective study of information quality in Canada, France and the United States. J Gen Intern Med. 2013. doi:10.1007/s11606-013-2411-7.

2. Light D. Pharmaceutical sales representatives and patient safety. J Gen Intern Med. 2013. doi:10.1007/s11606-013-2559-1.

3. Dormuth CR, Hemmelgarn BR, Paterson JM, et al. Use of high potency statins and rates of admission for acute kidney injury: multicenter, retrospective observational analysis of administrative databases. BMJ. 2013;346:f880. doi:10.1136/bmj.f880.

4. Therapeutics Initiative. High dose versus standard dose statins in stable coronary heart disease. Therapeutics Letter 87/Jul-Aug 2012. Available at: http://www.ti.ubc.ca/letter87. Accessed July 5, 2103

5. Turner EH, Matthews AM, Linardatos E, et al. Selective publication of antidepressant trials and its influence on apparent efficacy. N Engl J Med. 2008;358:252-60. 